\title{
Glucose and Lipid Metabolism Abnormalities among Patients with Autosomal Dominant Polycystic Kidney Disease
}

\author{
Magda Fliszkiewicz Mariusz Niemczyk Andrzej Kulesza Anna Łabuś \\ Leszek Pączek \\ Department of Immunology, Transplant Medicine, and Internal Diseases, Medical University \\ of Warsaw, Warsaw, Poland
}

\section{Keywords}

Autosomal dominant polycystic kidney disease $\cdot$ Diabetes mellitus $\cdot$ Kidney transplant

\begin{abstract}
Introduction: Autosomal dominant polycystic kidney disease (ADPKD) is the most prevalent monogenic renal disease with a prevalence of 1:1,000 births and it is the 4th most common cause of dialysis-dependent end-stage renal disease (ESDR). Recent reports suggest an association between APDKD and metabolic derangements, particularly impaired glucose metabolism. Methods: In this cross-sectional study we analyzed data obtained from case records of 189 patients with ADPKD, including kidney transplant recipients, managed in an outpatient department. Results: The mean BMI was $25.4 \pm 3.9 ; 25.25$ before and 27.7 after transplantation. A fasting glucose level above $100 \mathrm{mg} / \mathrm{dL}(5.6 \mathrm{mmol} / \mathrm{L})$ was observed in 60 patients (29\%) $-27 \%$ without transplantation and $41 \%$ kidney transplant recipients. Diabetes mellitus was diagnosed in 17 patients (8.9\%), including 3 (2.3\%) without a history of transplantation and $14(24.1 \%)$ after kidney transplantation $(p<0.01)$. We observed dyslipidemia in $30 \%$ and hyperuricemia in $53 \%$ of patients. Conclusion: Demonstrated metabolic abnormalities should be considered in maintenance of ADPKD patients, including kidney transplant recipients.
\end{abstract}

\footnotetext{
(C) 2019 The Author(s)

Published by S. Karger AG, Basel
}

\section{Introduction}

Autosomal dominant polycystic kidney disease (ADPKD) is the most prevalent monogenic renal disease with a prevalence of 1:1,000 births and it is the 4th most common cause of dialysis-dependent end-stage renal disease (ESDR) [1]. ADPKD manifests with kidney cysts 
leading to a progressive loss of the kidney function. It is caused by a mutation either in PKD1 (85\%) or PKD2 (15\%) gene, which encode polycystin-1 (PC1) and polycystin-2 (PC2), respectively [2]. These proteins form a transmembrane calcium channel complex, which can be found in various tissues, hence ADPKD can be regarded a systemic disease.

Recent reports suggest an association between APDKD and metabolic derangements, particularly impaired glucose metabolism. The character of these derangements is still unclear, while both increased insulin resistance [3] and impaired insulin release were observed [4]. The relationship between occurrence of new-onset diabetes after transplantation (NODAT) and ADPKD is not unambiguous, but recent meta-analyses do not exclude such a relation [5-7]. The incidence of posttransplant diabetes mellitus is connected with the type of immunosuppression and is higher among patients receiving calcineurin inhibitors, such as tacrolimus or cyclosporin (CsA) [8].

Hypercholesterolemia is frequently concomitant with ADPKD. According to the KHA-CARI guidelines [9] administration of statins should be considered in all patients with chronic kidney disease (CKD). Statins may slow the progression of cyst growth, especially administered in the early stage of the disease [10]. In the CRISP (Consortium for Radiologic Imaging Studies of Polycystic Kidney Disease) study a significant correlation between low high-density lipoprotein serum level and greater increase of total kidney volume was observed [11].

Metabolic derangements may accelerate the progression of ADPKD and cardiovascular complications. The aim of this study is to present metabolic abnormalities, especially concerning impaired fasting glucose and development of diabetes mellitus after transplantation, among patients with ADPKD in our outpatient clinic. Another aim is to assess whether metabolic derangements, including NODAT, differ depending on the type of immunosuppression in kidney transplant recipients with ADPKD.

\section{Materials and Methods}

In a cross-sectional study we analyzed data obtained from case records of 189 patients with ADPKD, including kidney transplant recipients, aged 18 years and older, managed in our outpatient department. In all participants, the diagnosis of ADPKD was established according to ultrasonographic criteria [12].

We assessed the following parameters: age, gender, kidney transplantation history, serum creatinine level, glomerular filtration rate (GFR), body mass index (BMI), presence of arterial hypertension, diabetes mellitus, fasting blood glucose level, cholesterol level, triglyceride level, uric acid level, and type of immunosuppressives. Considering the wide age range and higher occurrence of metabolic abnormalities in older patients, we selected two groups according to age, with the cutoff point at 50 years of age.

The biochemical assays were performed in the local hospital's central laboratory, using standard laboratory techniques with an autoanalyzer and immunochemical analyzer. The upper levels of the foregoing parameters, such as fasting glucose $(<100 \mathrm{mg} / \mathrm{dL},<5.6 \mathrm{mmol} / \mathrm{L})$, cholesterol ( $<200 \mathrm{mg} / \mathrm{dL})$, triglycerides $(<150 \mathrm{mg} / \mathrm{dL})$, and uric acid $(<6 \mathrm{mg} / \mathrm{dL})$ levels, was defined according to current ESC, EASD, and EULAR guidelines [13-16]. The estimated glomerular filtration rate (eGFR) was established using the CKD-EPI formula.

Parameters were compared between the study and the control group with the MannWhitney test for quantitative and with the Student $t$ test for qualitative variables. Results with $p<0.05$ were considered as statistically significant.

The study was performed with the principles of ethics. Due to the characteristic of the study, the consent of the bioethical commission as well as informed consent of the participants were not obligatory. 


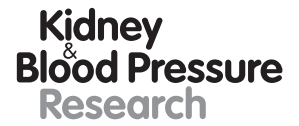

\begin{tabular}{l|l}
\hline Kidney Blood Press Res 2019;44:1416-1422 \\
\hline DOI: 10.1159/000503423 & $\begin{array}{l}\text { @ 2019 The Author(s). Published by S. Karger AG, Basel } \\
\text { www.karger.com/kbr }\end{array}$ \\
\hline
\end{tabular}

Fliszkiewicz et al.: Metabolic Abnormalities in ADPKD

\section{Results}

Our current study comprised 189 patients (83 men, 106 women), with an age range between 19 and 83 years and a mean age of $50.1 \pm 15.1$ years. 77 patients were under and 111 above 50 years of age. Among them, 58 patients (30\%) were kidney transplant recipients. The mean eGRF was $51.09 \pm 29.5 \mathrm{~mL} / \mathrm{min} / 1.73 \mathrm{~m}^{2}$ (range $3.5-131.1 \mathrm{~mL} / \mathrm{min} / 1.73 \mathrm{~m}^{2}$ ). Arterial hypertension was diagnosed in 166 patients (87.8\%).

The mean BMI was $25.4 \pm 3.9 ; 25.25$ before and 27.7 after transplantation. No statistically significant difference was observed. Among all of the participants 93 patients (49.2\%) had normal body weight with a BMI lower than 25,65 patients (34.3\%) had a BMI between 25 and 30 , and 31 patients $(16.4 \%$ ) were severely obese with a BMI higher than 30 , according to WHO guidelines [17]. We observed no significant difference in BMI in men and women. The mean BMI in the younger group was 24.9 , and it was 25.7 in the group older than 50 years. No significant difference between age groups was observed.

The mean fasting glucose level was $97.2 \pm 18 \mathrm{mg} / \mathrm{dL}(5.4 \pm 1 \mathrm{mmol} / \mathrm{L}), 90.19 \pm 8.5 \mathrm{mg} /$ $\mathrm{dL}(5.01 \pm 0.47 \mathrm{mmol} / \mathrm{L})$ in the group under 50 years of age, and $102.09 \pm 21.6 \mathrm{mg} / \mathrm{dL}$ (5.67 $\pm 1.2 \mathrm{mmol} / \mathrm{L})$ in the older group, respectively. In both groups no significant difference between patients without a history of transplantation and kidney transplant recipients was observed. A fasting glucose level above $100 \mathrm{mg} / \mathrm{dL}$ ( $5.6 \mathrm{mmol} / \mathrm{L}$ ) was observed in 60 patients (29\%) - 27\% without transplantation and $41 \%$ kidney transplant recipients. We observed no significant difference in fasting glucose level between patients without a history of transplantation and kidney transplant recipients as well as between genders. Furthermore, 17 patients (8.9\%) were diagnosed with diabetes mellitus, including $3(2.3 \%)$ without a history of transplantation and $14(24.1 \%)$ after kidney transplantation. The difference was statistically significant $(p=0.01)$. Additionally, we observed a strong positive correlation between BMI and fasting glucose level $(p<0.01)$. Interestingly, in all cases diabetes mellitus occurred in patients older than 50 years.

The mean serum cholesterol level was $183.4 \pm 39.12 \mathrm{mg} / \mathrm{dL}$ (range 102-298 mg/dL), 57 patients (30\%) had an elevated serum cholesterol level above $200 \mathrm{mg} / \mathrm{dL}-26 \%$ before and $41 \%$ after transplantation. Interestingly, women had higher cholesterol levels compared to men, and the difference was statistically significant $(p<0.01)$. The mean triglyceride level was $133.8 \pm 73.1 \mathrm{mg} / \mathrm{dL}, 30 \%$ of patients had elevated triglycerides above $150 \mathrm{mg} / \mathrm{dL}, 24 \%$ before and $41 \%$ after transplantation. There was no difference in the triglyceride level between men and women. In addition, 38\% of patients were treated with 3-hydroxy-3-methyl-glutaryl-CoA reductase inhibitors (statins) before transplantation and 50\% after transplantation.

The mean uric acid serum level was $6.1 \pm 1.6 \mathrm{mg} / \mathrm{dL}, 5.7 \mathrm{mg} / \mathrm{dL}$ before and $7.0 \mathrm{mg} / \mathrm{dL}$ after kidney transplantation. The difference was significant $(p<0.01)$. However, $53 \%$ of patients had an elevated serum uric acid level regardless of treatment with allopurinol.

Anthropometric and biochemical characteristics of the ADPKD patients before and after kidney transplantation are presented in Tables 1 and 2.

Among 58 kidney transplant recipients, 40 received tacrolimus-based immunosuppression and in 17 patients CsA-based immunosuppression was administered. All patients received steroids. We excluded from statistical analysis 1 patient who received azathioprine, a purine analogue belonging to the antimetabolic family. We observed a significant difference $(p<0.01)$ in mean BMI: $24.87 \pm 3.7$ in the tacrolimus group and $28.15 \pm 3.4$ in the CsA group. Interestingly, we observed no statistically significant difference in the occurrence of diabetes mellitus in both groups, with 9 patients $(22.5 \%)$ in the tacrolimus group and 5 patients $(29.4 \%)$ in the CsA group. As previously mentioned, all patients with diabetes mellitus were over 50 years of age. We observed no statistically significant difference in mean fasting glucose and serum cholesterol in both groups. 
Table 1. Anthropometric and biochemical characteristics of the ADPKD patients before and after kidney transplantation

\begin{tabular}{lcc}
\hline Parameter & $\begin{array}{l}\text { ADPKD } \\
(n=131)\end{array}$ & $\begin{array}{l}\text { ADPKD - kidney transplant } \\
\text { recipients }(n=58)\end{array}$ \\
\hline Age, years & $47.7 \pm 16.0$ & $56.8 \pm 10.2$ \\
Gender (male), $n(\%)$ & $55(41)$ & $28(48)$ \\
BMI & $25.25 \pm 4.0$ & $27.74 \pm 3.9$ \\
Fasting glucose, mg/dL & $95.1 \pm 14.1$ & $102.0 \pm 24.9$ \\
Diabetes, $n$ (\%) & $3(2.3)$ & $14(24.13)$ \\
Serum creatinine, mg/dL & $2.1 \pm 2.1$ & $1.7 \pm 0.8$ \\
Arterial hypertension, $n(\%)$ & $110(83)$ & $56(96)$ \\
Cholesterol, mg/dL & $137.9 \pm 38.1$ & $188.5 \pm 40.6$ \\
Triglycerides, mg/dL & $119.8 \pm 62.1$ & $111.9 \pm 85.1$ \\
Uric acid, $\mathrm{mg} / \mathrm{dL}$ & $5.7 \pm 1.6$ & $7.0 \pm 1.4$ \\
\hline
\end{tabular}

ADPKD, autosomal dominant polycystic kidney disease; BMI, body mass index.

Table 2. Anthropometric and biochemical characteristics of the ADPKD patients

\begin{tabular}{lc}
\hline Parameter & $\begin{array}{c}\text { Percentage of patients } \\
\text { with APDKD }(n=189)\end{array}$ \\
\hline Overweight (BMI 25-30) & $34.3 \%(n=65)$ \\
Obesity (BMI $>30)$ & $16.4 \%(n=31)$ \\
Cholesterol level $>200 \mathrm{mg} / \mathrm{dL}$ & $30.1 \%(n=57)$ \\
Diabetes mellitus & $8.9 \%(n=17)$ \\
Fasting glucose $>100 \mathrm{mg} / \mathrm{dL}$ & $29.1 \%(n=60)$ \\
Uric acid level $>6 \mathrm{mg} / \mathrm{dL}$ & $53.9 \%(n=102)$ \\
\hline
\end{tabular}

ADPKD, autosomal dominant polycystic kidney disease; BMI, body mass index.

\section{Discussion}

As previously mentioned, ADPKD is connected with various metabolic abnormalities. Probably the most important in the acceleration of cyst growth is impaired glucose metabolism, and certain mechanisms were identified, for example, insulin-like growth factor-1 (IGF-1) levels are high in obesity and increase proportionately with serum insulin level [18]. It was observed that IGF-1 plays a role in mediating tubular cell proliferation in the cystic kidney. Thereby, IGF-1 expression is responsible for ADPKD development [19]. This may have practical implications. In a small study, Pisani et al. [20] showed a slower decrease of GFR among diabetic patients with ADPKD treated with metformin compared to nondiabetic patients with APDKD.

In our study, we observed diabetes mellitus in $2.3 \%$ of patients with ADPKD and without a history of transplantation. Compared to the prevalence of $8.3 \%$ in the general population [21], the prevalence in our study is low. Furthermore, we observed diabetes mellitus in $24.1 \%$ of kidney transplant recipients. In a large study, Cosio et al. [22] showed that the incidence of NODAT among non-diabetes mellitus renal allograft recipients was $21 \% 10$ years after transplantation. Our study confirms that ADPKD may be a risk factor for NODAT [23]. Furthermore, all diabetes mellitus patients were older than 50 years. Interestingly, we observed no significant difference between the mean fasting glucose level in patients with ADPKD and no history 


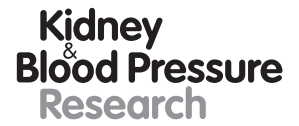

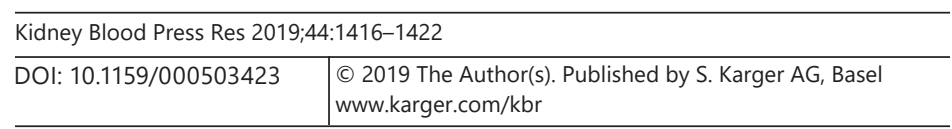

Fliszkiewicz et al.: Metabolic Abnormalities in ADPKD

of transplantation and kidney transplant recipients. We believe that kidney transplant recipients due to ADPKD should be particularly supervised and educated on diabetes prevention.

We demonstrated that $50.7 \%$ of APKDP patients had a BMI over 25 and according to WHO guidelines [17] this result can be qualified as overweight and obesity. BMI was higher among kidney transplant recipients. Similar to the general population, BMI in ADPKD individuals has been increasing, with a mean BMI of 26.57 between 1991 and 2011 (612 participants) [24]. In a recent study, Nowak et al. [25] showed that overweight and obesity are associated with faster progression, especially in the early stage of ADPKD. In a foregoing study, $56.4 \%$ of participants were overweight or obese and it was connected with greater annual growth of total kidney volume up to $9.4 \%$ compared to $6.1 \%$ in individuals with normal body weight. Therefore, patients with ADPKD should be encouraged to maintain normal body weight, particularly in the early stage of the disease, and the importance of adequate calorie intake should be pointed out in guidelines.

The importance of hypercholesterolemia and influence on progression of ADPKD is suggested, both in KDIGO [26] and KHA-CARI [9] Guidelines. Our research confirms the existence of impaired lipid metabolism, as $30 \%$ of APDKD patients had hypercholesterolemia and hypertriglyceridemia in comparison to the non-ADPKD CKD population in which the prevalence varied between 25 and 100\%, depending on the concomitant nephrotic syndrome [27, 28]. Among kidney transplant recipients, the overall prevalence of dyslipidemia is above $50 \%$ [29] compared to $41 \%$ in our study.

Interestingly, neither of the foregoing guidelines concerning ADPKD accentuate treatment of hyperuricemia, which is an independent risk factor for progression of CKD [30]. We observed elevated serum uric acid levels in 53\% of patients, regardless of treatment with allopurinol. Han et al. [31] found hyperuricemia in 23\% of ADPKD patients and showed an association between hyperuricemia and a faster annual decline in eGFR.

Additionally, we compared metabolic features in kidney transplant recipients depending on the type of immunosuppression. Since azathioprine has no influence on glucose metabolism, we assessed only patients receiving calcineurin inhibitors. We observed no difference in occurrence of diabetes mellitus between tacrolimus-based and CsA-based immunosuppression. This observation is in contradiction to a large study conducted by Torres et al. [32], where a higher risk of NODAT was observed in the tacrolimus-based immunosuppression group. Furthermore, we observed higher a BMI in the CsA-based immunosuppression group and the difference was statistically significant. Torres et al. observed a similar result, but without statistical significance. However, the limitation of our study is a relatively small group.

We demonstrated the coexistence of metabolic changes and ADPKD. Regarding inconsistent results of recent studies, concerning the nature of these derangements, additional studies are necessary.

\section{Conclusion}

Physicians treating patients with ADPKD should be aware of the incidence of metabolic abnormalities in this disease and advise non-drug treatment and education, especially to prevent overweight and obesity, and in case of kidney transplant recipients also diabetes mellitus prevention.

\section{Statement of Ethics}

The authors have no ethical conflicts to disclose. 
Kidney
Blood Pressure
Research

\begin{tabular}{l|l}
\hline Kidney Blood Press Res 2019;44:1416-1422 \\
\hline DOI: 10.1159/000503423 & $\begin{array}{l}\text { ○ 2019 The Author(s). Published by S. Karger AG, Basel } \\
\text { www.karger.com/kbr }\end{array}$ \\
\hline
\end{tabular}

Fliszkiewicz et al.: Metabolic Abnormalities in ADPKD

\section{Disclosure Statement}

The authors have no conflicts of interest to declare.

\section{Funding Sources}

The authors did not receive any funding.

\section{Author Contributions}

Magda Fliszkiewicz: design of the study, data collection and analysis, literature search, writing the manuscript, final approval (50\%). Mariusz Niemczyk: design of the study, analysis of data, writing the manuscript, final approval (20\%). Andrzej Kulesza: analysis of data, final approval (10\%). Anna Łabuś: analysis of data, final approval (10\%). Leszek Pqczek: analysis of data, final approval (10\%).

\section{References}

1 Rangan GK, Tchan MC, Tong A, Wong AT, Nankivell BJ. Recent advances in autosomal-dominant polycystic kidney disease. Intern Med J. 2016 Aug;46(8):883-92.

2 Torres VE, Harris PC, Pirson Y. Autosomal dominant polycystic kidney disease. Lancet. 2007 Apr; 369(9569): 1287-301.

3 Vareesangthip K, Tong P, Wilkinson R, Thomas TH. Insulin resistance in adult polycystic kidney disease. Kidney Int. 1997 Aug;52(2):503-8.

4 Pietrzak-Nowacka M, Safranow K, Byra E, Nowosiad M, Marchelek-Myśliwiec M, Ciechanowski K. Glucose metabolism parameters during an oral glucose tolerance test in patients with autosomal dominant polycystic kidney disease. Scand J Clin Lab Invest. 2010 Dec;70(8):561-7.

5 Mao Z, Xie G, Ong AC. Metabolic abnormalities in autosomal dominant polycystic kidney disease. Nephrol Dial Transplant. 2015 Feb;30(2):197-203.

6 Yang B, Chen S, Yang G, Mei C, Ong A, Mao Z. New onset diabetes after kidney transplantation in patients with autosomal dominant polycystic kidney disease: systematic review protocol. BMJ Open. 2015 Nov;5(11): e008440.

7 Cheungpasitporn W, Thongprayoon C, Vijayvargiya P, Anthanont P, Erickson SB. The Risk for New-Onset Diabetes Mellitus after Kidney Transplantation in Patients with Autosomal Dominant Polycystic Kidney Disease: A Systematic Review and Meta-Analysis. Can J Diabetes. 2016 Dec;40(6):521-8.

8 Fonseca AC, Carvalho E, Eriksson JW, Pereira MJ. Calcineurin is an important factor involved in glucose uptake in human adipocytes. Mol Cell Biochem. 2018 Aug;445(1-2):157-68.

9 Mallett A, Patel M, Tunnicliffe DJ, Rangan GK. KHA-CARI Autosomal Dominant Polycystic Kidney Disease Guideline: Management of Renal Stone Disease. Semin Nephrol. 2015 Nov;35(6):603-606.e3.

10 Ecder T. Statins in the treatment of autosomal dominant polycystic kidney disease. Nephrol Dial Transplant. 2016 Aug;31(8):1194-6.

11 Torres VE, Grantham JJ, Chapman AB, Mrug M, Bae KT, King BF Jr, et al.; Consortium for Radiologic Imaging Studies of Polycystic Kidney Disease (CRISP). Potentially modifiable factors affecting the progression of autosomal dominant polycystic kidney disease. Clin J Am Soc Nephrol. 2011 Mar;6(3):640-7.

12 Pei Y, Obaji J, Dupuis A, Paterson AD, Magistroni R, Dicks E, et al. Unified criteria for ultrasonographic diagnosis of ADPKD. J Am Soc Nephrol. 2009 Jan;20(1):205-12.

13 Rydén L, Grant PJ, Anker SD, Berne C, Cosentino F, Danchin N, et al.; Document Reviewers. ESC Guidelines on diabetes, pre-diabetes, and cardiovascular diseases developed in collaboration with the EASD: the Task Force on diabetes, pre-diabetes, and cardiovascular diseases of the European Society of Cardiology (ESC) and developed in collaboration with the European Association for the Study of Diabetes (EASD). Eur Heart J. 2013 Oct;34(39):3035-87.

14 Catapano AL, Graham I, De Backer G, Wiklund O, Chapman MJ, Drexel H, et al. 2016 ESC/EAS Guidelines for the Management of Dyslipidaemias. Rev Esp Cardiol (Engl Ed). 2017 Feb;70(2):115.

15 Mancia G, Fagard R, Narkiewicz K, Redon J, Zanchetti A, Böhm M, et al.; Task Force for the Management of Arterial Hypertension of the European Society of Hypertension and the European Society of Cardiology. 2013 ESH/ESC Practice Guidelines for the Management of Arterial Hypertension. Blood Press. 2014 Feb;23(1): 3-16. 
Richette P, Doherty M, Pascual E, Barskova V, Becce F, Castañeda-Sanabria J, et al. 2016 updated EULAR evidence-based recommendations for the management of gout. Ann Rheum Dis. 2017 Jan;76(1):29-42. hensive version which includes systematic evidence review, evidence statements, and recommendations). Obesity (Silver Spring). 2014 Jul;22(S2 Suppl 2):S40.

18 Lewitt MS, Dent MS, Hall K. The Insulin-Like Growth Factor System in Obesity, Insulin Resistance and Type 2 Diabetes Mellitus. J Clin Med. 2014 Dec;3(4):1561-74.

19 Liu C, Zhang Y, Yuan L, Fu L, Mei C. Rosiglitazone inhibits insulin-like growth factor-1-induced polycystic kidney disease cell growth and p70S6 kinase activation. Mol Med Rep. 2013 Sep;8(3):861-4.

20 Pisani A, Riccio E, Bruzzese D, Sabbatini M. Metformin in autosomal dominant polycystic kidney disease: experimental hypothesis or clinical fact? BMC Nephrol. 2018 Oct;19(1):282.

21 Forouhi NG, Wareham NJ. Epidemiology of diabetes. Medicine (Abingdon). 2014 Dec;42(12):698-702.

22 Cosio FG, Pesavento TE, Osei K, Henry ML, Ferguson RM. Post-transplant diabetes mellitus: increasing incidence in renal allograft recipients transplanted in recent years. Kidney Int. 2001 Feb;59(2):732-7.

23 Hamer RA, Chow CL, Ong AC, McKane WS. Polycystic kidney disease is a risk factor for new-onset diabetes after transplantation. Transplantation. 2007 Jan;83(1):36-40.

24 Helal I, McFann K, Reed B, Yan XD, Schrier RW. Changing referral characteristics of patients with autosomal dominant polycystic kidney disease. Am J Med. 2013 Sep;126(9):832.e7-11.

25 Nowak KL, You Z, Gitomer B, Brosnahan G, Torres VE, Chapman AB, et al. Overweight and Obesity Are Predictors of Progression in Early Autosomal Dominant Polycystic Kidney Disease. J Am Soc Nephrol. 2018 Feb;29(2):571-8.

26 Chapman AB, Devuyst O, Eckardt KU, Gansevoort RT, Harris T, Horie S, et al.; Conference Participants. Autosomal-dominant polycystic kidney disease (ADPKD): executive summary from a Kidney Disease: Improving Global Outcomes (KDIGO) Controversies Conference. Kidney Int. 2015 Jul;88(1):17-27.

27 Kasiske BL. Hyperlipidemia in patients with chronic renal disease. Am J Kidney Dis. 1998 Nov;32(5 Suppl 3):S142-56.

28 Wanner C, Tonelli M; Kidney Disease: Improving Global Outcomes Lipid Guideline Development Work Group Members. KDIGO Clinical Practice Guideline for Lipid Management in CKD: summary of recommendation statements and clinical approach to the patient. Kidney Int. 2014 Jun;85(6):1303-9.

29 Kidney Disease: Improving Global Outcomes (KDIGO) Transplant Work Group. KDIGO clinical practice guideline for the care of kidney transplant recipients. Am J Transplant. 2009 Nov; 9 Suppl 3:S1-155.

30 Levy G, Shi JM, Cheetham TC, Rashid N. Urate-Lowering Therapy in Moderate to Severe Chronic Kidney Disease. Perm J. 2018;22:17-142.

31 Han M, Park HC, Kim H, Jo HA, Huh H, Jang JY, et al. Hyperuricemia and deterioration of renal function in autosomal dominant polycystic kidney disease. BMC Nephrol. 2014 Apr;15(1):63.

32 Torres A, Hernández D, Moreso F, Serón D, Burgos MD, Pallardó LM, et al. Randomized Controlled Trial Assessing the Impact of Tacrolimus Versus Cyclosporine on the Incidence of Posttransplant Diabetes Mellitus. Kidney Int Rep. 2018 Jul;3(6):1304-15. 